of the simple isatin or ninhydrin reactions. It is entirely specific for hydroxy-proline among more than a hundred and twenty amino-acids, amines and heterocyclic acids tested.

We suggest that when new location reagents are examined, the extent to which they may be used in the ways outlined here should be investigated.

JOHN B. JEPSON

Courtauld Institute of Biochemistry, IVOR SMITH

Middlesex Hospital Medical School, London, W.1.

Oct. 27.

' Dent; Biochem. J., 43, 169 (1948).

2 For example, (a) Toennies and Kolb, Anal. Chem., 23, 823 (1951) (b) Hausmann, J. Amer. Chem. Soc., 74, 3181 (1952). (c) Acher and Crocker, Biochim. Biophys. Acta, 9, 704 (1952).

smith, Nature, 171, 43 (1953).

- Sakaguchi, J. Biochem. (Japan), 37, 231 (1950).

sanger and Tuppy, Biochem. J., 49, 463 (1951).

\section{Effect of Surface Films on the Evaporation of Water}

By a variety of laboratory experiments, it has been demonstrated that the presence upon a water surface of a suitable monolayer or duplex film may diminish considerably the rate of evaporation ${ }^{1-3}$. That significant retardation of evaporation is not obtained under natural conditions is indicated by the lack of application of the techniques on a larger scale.

It has been stated ${ }^{4}$ that the spreading of monolayers does not provide a practicable tochnique for restricting natural evaporation, because the resistance to transport of vapour provided by the film usually is small compared with the resistance offered by the non-turbulent layer of air above the water surface. It should be noted, however, that the alternative procedure of spreading thick duplex films provides no actual improvement. Fluid duplex films revert to an equilibrium state comprising a monolayer in equilibrium with lenses of oil ${ }^{4}$, and in this form offer less resistance to the passage of water vapour than do monolayers of long-chain alcohols. Rigid duplex films are fractured readily by wind and dust.

In the present work, the intrinsic resistances of several monolayers and duplex films have been determined between $20^{\circ} \mathrm{C}$. and $60^{\circ} \mathrm{C}$., using a technique similar to that adopted by Langmuir and Schaefer ${ }^{2}$. The $\mathrm{C}_{16^{-}}$and $\mathrm{C}_{18}$-aliphatic alcohols provided the least permeable monolayers of those examined, the maximum rate of evaporation through aged films being about six hundred times less than that determined for a clear water surface ${ }^{1,5}$ at $25^{\circ} \mathrm{C}$. It has been ascertained that monolayers of these substances are affected but slightly by wind and dust.

Except for two periods of a few hours duration, the spreading of monolayers did not reduce significantly the natural rate of evaporation during intervals of the 1952-53 Melbourne summer. On both these occasions, strong desiccating winds were experienced late on dull afternoons. During periods of the 1953 Melbourne winter an average reduction of 25 per cent has been obtained. Marked deviations about this mean value have been observed from day to day, depending on the weather. Simulation in the laboratory of various meteorological conditions produces a parallel variation in the efficiency of monolayers.

The explanation lies in the heat exchange between water and its surroundings. The restriction of evaporation leads to an increase in surface temperature, and accordingly to a compensating increase in the rate of evaporation. Under normal summer conditions, the compensating effect is sufficient to destroy the potential efficacy of a film. In cloudy weather the amount of long-wave radiation reaching the water surface is reduced, and both the surface temperatures and the significance of the compensating effect are decreased also. Accordingly, evaporation is retarded to an extent depending on such conditions. Strong winds also are helpful. It has been both calculated and observed that the absolute rate of evaporation from a film-covered surface decreases as the wind velocity increases, provided the surface receives a flux of radiant energy corresponding to that available near noon on cloudless summer days.

It is apparent that the spreading of surface films cannot significantly reduce the natural rate of evaporation during summer unless some method of restricting the absorption of (particularly long-wave) radiation is provided also. Appropriate investigations are in progress.

Division of Industrial Chemistry,

W. W. Mansfield

Commonwealth Scientific and

Industrial Research Organization, Melbourne, Australia.

Aug. 25.

${ }^{1}$ Baranaev, M., J. Phys. Chem. U.S.S.R., 9, 69 (1937).

2 Langmuir, I., and Schaefer, V. J., J. Franklin Inst., 235, 119 (1943).

${ }^{3}$ Docking, A. R., Heymann, E., Kerley, L. F., and Mortensen, K. M., Nature, 146, 265 (1940).

4 Heymann, E., and Yoffe, A., Trans. Farad. Soc., 38, 408 (1942)

${ }^{5}$ Alty, T., Nature, 139, 374 (1937).

\section{Analytical Separation of the Methyl Esters of the $C_{12}-C_{22}$ Fatty Acids by Vapour- Phase Chromatography}

James and Martin ${ }^{1}$ separated the fatty acids up to lauric acid by passage through a column of kieselguhr and silicone DC.550/stearic acid at $137^{\circ} \mathrm{C}$., and measured each fraction automatically by alkali titration.

Mr. N. H. Ray (Imperial Chemical Industries, Ltd., Alkali Division, Winnington) has applied the method to a variety of the lower-boiling alcohols, esters, ketones and hydrocarbons, using dinonyl phthalate (on 'Celite $545^{\prime}$ ) as static liquid phase, and using catharometers and a high-speed recorder for detecting and measuring each component (private communication).

We have developed the technique of vapour-phase chromatography so that separation and measurement of the $\mathrm{C}_{12}-\mathrm{C}_{22}$ fatty acid esters is now possible, at $230^{\circ} \mathrm{C}$. The chromatographic column consists of a vertical spiral 1 in. in diameter with twelve turns (pitch $\frac{1}{2}$ in.), so that the overall length of the column is about $3 \mathrm{ft}$.; this is packed with the dry mixture of 'Celite' (Johns Manville Co., Ltd.) and Dow Corning High Vacuum Silicone Grease (equal parts, previously mixed by grinding). At the entrance to, and the exit from, the column are attached two platinum-wire catharometers which are connected in a Wheatstone bridge arrangement so that the difference in resistance can be recorded by a Cambridge high-speed recorder. The column and catharometers are wound with asbestos tape so as to form a single unit, which is then placed in an iron cylinder, wound and lagged so that temperatures up to $300^{\circ} \mathrm{C}$. (by adjustment of a 'Variac') can be attained. The 